\title{
A TWO-PARAMETER EXTENSION OF URBANIK'S PRODUCT CONVOLUTION SEMIGROUP
}

\author{
BY
}

CHRISTIAN BERG (COPENHAGEN)

\begin{abstract}
We prove that $s_{n}(a, b)=\Gamma(a n+b) / \Gamma(b), n=0,1, \ldots$, is an infinitely divisible Stieltjes moment sequence for arbitrary $a, b>0$. Its powers $s_{n}(a, b)^{c}, c>0$, are Stieltjes determinate if and only if $a c \leqslant 2$. The latter was conjectured in a paper by Lin (2019) in the case $b=1$. We describe a product convolution semigroup $\tau_{c}(a, b), c>0$, of probability measures on the positive half-line with densities $e_{c}(a, b)$ and having the moments $s_{n}(a, b)^{c}$. We determine the asymptotic behavior of $e_{c}(a, b)(t)$ for $t \rightarrow 0$ and for $t \rightarrow \infty$, and the latter implies the Stieltjes indeterminacy when $a c>2$. The results extend the previous work of the author and López (2015) and lead to a convolution semigroup of probability densities $\left(g_{c}(a, b)(x)\right)_{c>0}$ on the real line. The special case $\left(g_{c}(a, 1)(x)\right)_{c>0}$ are the convolution roots of the Gumbel distribution with scale parameter $a>0$. All the densities $g_{c}(a, b)(x)$ lead to determinate Hamburger moment problems.
\end{abstract}

2010 AMS Mathematics Subject Classification: Primary: 60E07; Secondary: 60B15, 44A60.

Key words and phrases: Infinitely divisible Stieltjes moment sequence, product convolution semigroup, asymptotic approximation of integrals, Gumbel distribution.

\section{INTRODUCTION}

A Stieltjes moment sequence is a sequence of non-negative numbers of the form

$$
s_{n}=\int_{0}^{\infty} t^{n} d \mu(t), \quad n \in \mathbb{N}_{0}:=\{0,1,2, \ldots\},
$$

where $\mu$ is a positive measure on $[0, \infty)$ such that $t^{n} \in L^{1}(\mu)$ for all $n \in \mathbb{N}_{0}$. The sequence $\left(s_{n}\right)$ is called normalized if $s_{0}=\mu([0, \infty))=1$, and it is called $S$ determinate (resp. $S$-indeterminate) if (‥D) has exactly one solution (resp. several solutions) $\mu$ as positive measures on $[0, \infty)$. All these concepts go back to the fundamental memoir of Stieltjes [19]. 
A Stieltjes moment sequence $\left(s_{n}\right)$ is called infinitely divisible if $\left(s_{n}^{c}\right)$ is a Stieltjes moment sequence for any $c>0$. These sequences were characterized in Tyan's $\mathrm{Ph} . \mathrm{D}$. thesis [21] and again in [5] without the knowledge of [21]. An important example of an infinitely divisible normalized Stieltjes moment sequence is $s_{n}=n$ !, first established in Urbanik [22]. He proved that $e_{c}$ in (1L.2) is a probability density such that

$$
(n !)^{c}=\int_{0}^{\infty} t^{n} e_{c}(t) d t, \quad e_{c}(t)=\frac{1}{2 \pi} \int_{-\infty}^{\infty} t^{i x-1} \Gamma(1-i x)^{c} d x, \quad c, t>0 .
$$

Here $\Gamma$ is Euler's gamma function. The family $\left(\tau_{c}\right)_{c>0}$ with $d \tau_{c}(t)=e_{c}(t) d t$ is a convolution semigroup in the sense of [6] on the locally compact abelian group $G=(0, \infty)$ under multiplication. It is called the Urbanik semigroup in [ $\square]$. It turns out that the terminology "Urbanik semigroup" has been used in the literature for certain semigroups of operators on Banach spaces with the precise name "Urbanik decomposability semigroup", see Section 2 in [10]. We have therefore decided to use the more precise name "Urbanik's product convolution semigroup".

By Carleman's criterion for S-determinacy it is easy to prove that $(n !)^{c}$ is $\mathbf{S}$ determinate for $c \leqslant 2$. That this estimate is sharp was first proved in [4], where it was established that $(n !)^{c}$ is S-indeterminate for $c>2$ based on asymptotic results of Skorokhod [18] about stable distributions, see [23]. Another proof of the Sindeterminacy was given in [ [] based on the asymptotic behavior of $e_{c}(t)$,

$$
e_{c}(t)=\frac{(2 \pi)^{(c-1) / 2}}{\sqrt{c}} \frac{\exp \left(-c t^{1 / c}\right)}{t^{(c-1) /(2 c)}}\left[1+\mathcal{O}\left(t^{-1 / c}\right)\right], \quad t \rightarrow \infty .
$$

In the recent paper [11] , Lin proposes the following conjecture:

Conjecture. Let $a>0$ be a real constant and let $s_{n}=\Gamma(n a+1), n \in \mathbb{N}_{0}$. Then:

(a) $\left(s_{n}\right)$ is an infinitely divisible Stieltjes moment sequence.

(b) For real $c>0$ the sequence $\left(s_{n}^{c}\right)$ is $S$-determinate if and only if ac $\leqslant 2$.

(c) For $0<c \leqslant 2 /$ a the unique probability measure $\mu_{c}$ corresponding to $\left(s_{n}^{c}\right)$ has the Mellin transform

$$
\int_{0}^{\infty} t^{s} d \mu_{c}(t)=\Gamma(a s+1)^{c}, \quad s \geqslant 0 .
$$

When $a=1$, the conjecture is true because of the known results about Urbanik's product convolution semigroup, and for $a \in \mathbb{N}, a \geqslant 2$, the conjecture is true because of Theorems 4 and 7 in [11].

We shall prove that the conjecture is true, and it is a special case of similar results for the following more general normalized Stieltjes moment sequence 


$$
s_{n}(a, b)=\frac{\Gamma(a n+b)}{\Gamma(b)}=\frac{1}{a \Gamma(b)} \int_{0}^{\infty} t^{n} t^{b / a-1} \exp \left(-t^{1 / a}\right) d t, \quad n=0,1, \ldots,
$$

where $a, b>0$ are arbitrary.

Defining

$$
e_{1}(a, b)(t)=\frac{1}{a \Gamma(b)} t^{b / a-1} \exp \left(-t^{1 / a}\right)
$$

we get for Re $z>-b / a$ and after a change of variable $t=s^{a}$

$$
\int_{0}^{\infty} t^{z} e_{1}(a, b)(t) d t=\Gamma(a z+b) / \Gamma(b)
$$

This leads to our first main result.

THEOREM 1.1. (i) $\left(s_{n}(a, b)\right)$ is an infinitely divisible Stieltjes moment sequence.

(ii) There exists a uniquely determined convolution semigroup $\left(\tau_{c}(a, b)\right)_{c>0}$ of probability measures on the multiplicative group $(0, \infty)$ such that

$$
\int_{0}^{\infty} t^{z} d \tau_{c}(a, b)(t)=[\Gamma(a z+b) / \Gamma(b)]^{c}, \quad \operatorname{Re} z>-b / a,
$$

and, in particular, $\left(s_{n}(a, b)^{c}\right)$ is the moment sequence of $\tau_{c}(a, b)$.

(iii) $d \tau_{c}(a, b)(t)=e_{c}(a, b)(t) d t$ on $(0, \infty)$, where

$$
e_{c}(a, b)(t)=\frac{1}{2 \pi} \int_{-\infty}^{\infty} t^{i x-1}[\Gamma(b-i a x) / \Gamma(b)]^{c} d x, \quad t>0,
$$

is a probability density belonging to $C^{\infty}(0, \infty)$.

(iv) $\left(s_{n}(a, b)^{c}\right)$ is $S$-determinate if and only if ac $\leqslant 2$, hence independent of $b>0$.

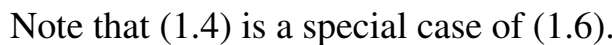

The measure $\tau_{1}(a, b)$ was considered in [20], where it was proved that the measure is $\mathrm{S}$-indeterminate if $a>\max (2,2 b)$. This is a consequence of our result. Note that $\tau_{1}(a, 1)$ is called the Weibull distribution with shape parameter $1 / a$ and scale parameter one.

In ([L]) and ([L.8) we use that $\Gamma(z)$ is a non-vanishing holomorphic function in the cut plane

$$
\mathcal{A}=\mathbb{C} \backslash(-\infty, 0],
$$


so we can define

$$
\Gamma(z)^{c}:=\exp (c \log \Gamma(z)), \quad z \in \mathcal{A},
$$

using the holomorphic branch of $\log \Gamma$ which is zero for $z=1$. This branch is explicitly given in (B.1) below.

Let us recall a few facts about convolution semigroups of probability measures on LCA groups, see [6] for details.

The continuous characters of the multiplicative group $G=(0, \infty)$ can be given as $t \rightarrow t^{i x}$, where $x \in \mathbb{R}$ is arbitrary, and in this way the dual group $\widehat{G}$ of $G$ can be identified with the additive group of real numbers. The convolution between measures $\mu$ and $\sigma$ on $(0, \infty)$, called a product convolution and denoted by $\mu \diamond \sigma$, is defined as

$$
\int_{0}^{\infty} f(t) d \mu \diamond \sigma(t)=\int_{0}^{\infty} \int_{0}^{\infty} f(t s) d \mu(t) d \sigma(s)
$$

for suitable classes of continuous functions $f$ on $(0, \infty)$, e.g. those of compact support.

A family $\left(\mu_{c}\right)_{c>0}$ of probability measures on the multiplicative group $G=$ $(0, \infty)$ is called a convolution semigroup if

$$
\mu_{c} \diamond \mu_{d}=\mu_{c+d}, c, d>0, \quad \text { and } \quad \lim _{c \rightarrow 0} \mu_{c}=\varepsilon_{1} \text { vaguely. }
$$

Here $\varepsilon_{1}$ is the Dirac measure with total mass one concentrated in the neutral element one of the group. Given a convolution semigroup $\left(\mu_{c}\right)_{c>0}$ on $(0, \infty)$, it is easy to see that if $\mu_{1}$ has moments of order $n$, then all the measures $\mu_{c}$ have moments of order $n$ and

$$
\int_{0}^{\infty} t^{n} d \mu_{c}(t)=\left(\int_{0}^{\infty} t^{n} d \mu_{1}(t)\right)^{c}, \quad c>0
$$

By [6], Theorem 8.3, there is a one-to-one correspondence between convolution semigroups $\left(\mu_{c}\right)_{c>0}$ of probability measures on $G$ and continuous negative definite functions $\rho: \mathbb{R} \rightarrow \mathbb{C}$ satisfying $\rho(0)=0$ such that

$$
\int_{0}^{\infty} t^{-i x} d \mu_{c}(t)=\exp (-c \rho(x)), \quad c>0, x \in \mathbb{R} .
$$

By the inversion theorem of Fourier analysis for LCA groups, if $\exp (-c \rho)$ is integrable on $\mathbb{R}$, then $d \mu_{c}(t)=f_{c}(t) d t$ for a continuous function $f_{c}(t)\left(t f_{c}(t)\right.$ is the density of $\mu_{c}$ with respect to Haar measure $(1 / t) d t$ on $\left.(0, \infty)\right)$ given by

$$
f_{c}(t)=\frac{1}{2 \pi} \int_{-\infty}^{\infty} t^{i x-1} \exp (-c \rho(x)) d x, \quad t>0 .
$$

(Note that the dual Haar measure of $(1 / t) d t$ on $(0, \infty)$ is $1 /(2 \pi) d x$ on $\mathbb{R}$.) 
Proposition 1.1. For $a, b>0$

$$
\rho(x):=\log \Gamma(b)-\log \Gamma(b-i a x), \quad x \in \mathbb{R},
$$

is a continuous negative definite function on $\mathbb{R}$ satisfying $\rho(0)=0$.

Proposition $\mathbb{L}]$ shows that there exists a uniquely determined product convolution semigroup $\left(\tau_{c}(a, b)\right)_{c>0}$ satisfying

$$
\begin{aligned}
\int_{0}^{\infty} t^{-i x} d \tau_{c}(a, b)(t) & =\exp [-c(\log \Gamma(b)-\log \Gamma(b-i a x))] \\
& =[\Gamma(b-i a x) / \Gamma(b)]^{c}, \quad x \in \mathbb{R} .
\end{aligned}
$$

Putting $z=-i x$ in (ㄷ.6), we see by the uniqueness theorem for Fourier transforms that $d \tau_{1}(a, b)(t)=e_{1}(a, b)(t) d t$, and since $e_{1}(a, b)(t)$ has moments of any order by ([.6), we infer that all the measures $\tau_{c}(a, b)$ have moments of any order. This implies that the integral

$$
\int_{0}^{\infty} t^{z} d \tau_{c}(a, b)(t), \quad \operatorname{Re} z \geqslant 0,
$$

defines a continuous function of $z$ in the half-plane $\operatorname{Re} z \geqslant 0$ and holomorphic in the interior $\operatorname{Re} z>0$. By $\left([\cdot]_{3}\right)$ this function equals $[\Gamma(b+a z) / \Gamma(b)]^{c}$ on the imaginary axis and hence on $\operatorname{Re} z \geqslant 0$. As in the proof of [4], Lemma 2.1, it follows that this equality extends to the half-plane $\operatorname{Re} z>-b / a$, i.e. (IL.7) holds.

The function $(\Gamma(b-i a x) / \Gamma(b))^{c}$ is a Schwartz function on $\mathbb{R}$ and in particular

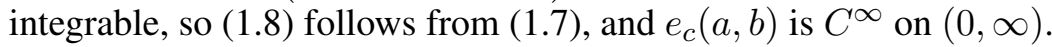

In this way we have established (i)-(iii) of Theorem $\mathrm{L}$. . The proof of the more difficult part (iv) as well as the proof of Proposition $1 \mathrm{l}$. will be given in Section 3.

By Riemann-Lebesgue's lemma we also see that $t e_{c}(a, b)(t)$ tends to zero for $t$ tending to zero and to infinity. Much more on the behavior near zero and infinity will be given in Section 2, where we extend the work of [ $⿴ 囗 十$ ] leading to the asymptotic behavior of the densities $e_{c}(a, b)(t)$ for $t \rightarrow 0$ and $t \rightarrow \infty$. The behavior for $t \rightarrow \infty$ will lead to a proof of the S-indeterminacy for $a c>2$ using the Krein criterion.

The fact that $\tau_{c}(a, b) \diamond \tau_{d}(a, b)=\tau_{c+d}(a, b)$ can be written as

$$
e_{c+d}(a, b)(t)=\int_{0}^{\infty} e_{c}(a, b)(t / x) e_{d}(a, b)(x) \frac{d x}{x}, \quad c, d>0 .
$$

In particular, for $c=d=1$ and the explicit formula for $e_{1}(a, b)$ we get

$$
\begin{aligned}
e_{2}(a, b)(t) & =\frac{t^{b / a-1}}{[a \Gamma(b)]^{2}} \int_{0}^{\infty} \exp \left(-x^{-1 / a} t^{1 / a}-x^{1 / a}\right) \frac{d x}{x} \\
& =\frac{2 t^{b / a-1}}{a \Gamma(b)^{2}} K_{0}\left(2 t^{1 /(2 a)}\right)
\end{aligned}
$$


because the Macdonald function $K_{0}$ is given by

$$
K_{0}(z)=\frac{1}{2} \int_{0}^{\infty} \exp \left(-(z / 2)^{2} / y-y\right) \frac{d y}{y}
$$

cf. [8], 8.432(6); [14], Chapter 10, Section 25.

Except for a scaling this result is the same as Lemma 1 in [12].

\section{MAIN RESULTS}

Our additional main results are the following:

THEOREM 2.1. For $c>0$ we have

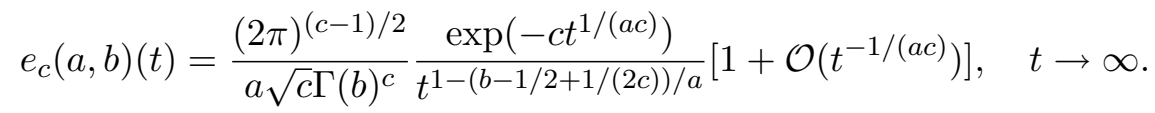

THEOREM 2.2. The measure $\tau_{c}(a, b)$ is $S$-indeterminate if and only if $a c>2$.

THEOREM 2.3. For $c>0$ and $0<t<1$ we have

$$
e_{c}(a, b)(t)=\frac{t^{b / a-1}}{[a \Gamma(b)]^{c}} \frac{[\log (1 / t)]^{c-1}}{\Gamma(c)}+\mathcal{O}\left(t^{b / a-1}[\log (1 / t)]^{c-2}\right), \quad t \rightarrow 0 .
$$

REMARK 2.1. Formula (2.2) shows that $e_{c}(a, b)(t)$ tends to zero for $t \rightarrow 0$ if $b / a>1$, and to infinity if $b / a<1$, independent of $c$. If $b / a=1$, then $e_{c}(a, b)(t)$ tends to zero for $c<1$ and to infinity as a power of $\log (1 / t)$ when $c>1$.

\section{PROOFS}

Pro of of Proposition $\mathbb{L}$. From the Weierstrass product for the entire function $1 / \Gamma(z)$ we get the following holomorphic branch in the cut plane $\mathcal{A}$, cf. (ㅍ. $)$ :

$$
-\log \Gamma(z)=\gamma z+\log z+\sum_{k=1}^{\infty}(\log (1+z / k)-z / k), \quad z \in \mathcal{A},
$$

where $\log$ denotes the principal logarithm, and $\gamma$ is Euler's constant.

For $n \in \mathbb{N}$ and $z \in \mathcal{A}$ define

$$
\begin{gathered}
\rho_{n}(z)=\gamma z+\log z+\sum_{k=1}^{n}(\log (1+z / k)-z / k), \\
R_{n}(z)=\sum_{k=n+1}^{\infty}(\log (1+z / k)-z / k),
\end{gathered}
$$


so $\lim _{n \rightarrow \infty} \rho_{n}(z)=-\log \Gamma(z)$, uniformly on compact subsets of $\mathcal{A}$. Furthermore, we have

$$
\log \Gamma(b)+\rho_{n}(b)+R_{n}(b)=0,
$$

and since $\log (1+x)<x$ for $x>0$, we see that $R_{n}(b)<0$ and hence $\log \Gamma(b)+$ $\rho_{n}(b)>0$.

We claim that $\log \Gamma(b)+\rho_{n}(b-i a x)$ is a continuous negative definite function, and letting $n \rightarrow \infty$, we get the assertion of Proposition $\mathbb{L}$.

To see the claim, we write

$$
\begin{aligned}
\log \Gamma(b)+\rho_{n}(b-i a x)= & \log \Gamma(b)+(b-i a x)\left(\gamma-\sum_{k=1}^{n} \frac{1}{k}\right) \\
& +\log (b-i a x)+\sum_{k=1}^{n} \log \left(1+\frac{b-i a x}{k}\right) \\
=\log \Gamma(b)+\rho_{n}(b) & -i a x\left(\gamma-\sum_{k=1}^{n} \frac{1}{k}\right)+\sum_{k=0}^{n} \log \left(1-i \frac{a x}{b+k}\right),
\end{aligned}
$$

and the assertion follows since $\alpha+i \beta x$ and $\log (1+i \beta x)$ are negative definite functions when $\alpha \geqslant 0, \beta \in \mathbb{R}$, see [6], [16].

Pro of of The or e m [2.1. We modify the proof given in [7] and start by applying Cauchy's integral theorem to move the integration in ([L.8) to a horizontal line

$$
H_{\delta}:=\{z=x+i \delta: x \in \mathbb{R}\}, \quad \delta>-b / a .
$$

LEMMA 3.1. With $H_{\delta}$ as in (B.2) we have

$$
e_{c}(a, b)(t)=\frac{1}{2 \pi} \int_{H_{\delta}} t^{i z-1}[\Gamma(b-i a z) / \Gamma(b)]^{c} d z, \quad t>0 .
$$

Proof. For $t, c>0$ fixed, $f(z)=t^{i z-1}[\Gamma(b-i a z) / \Gamma(b)]^{c}$ is holomorphic in the simply connected domain $\mathbb{C} \backslash i(-\infty,-b / a]$, so (3.3) follows from Cauchy's integral theorem provided the integral

$$
\int_{0}^{\delta} f(x+i y) d y
$$

tends to zero for $x \rightarrow \pm \infty$. We have

$$
|f(x+i y)|=t^{-y-1}|\Gamma(b+y-i a x) / \Gamma(b)|^{c},
$$

and the result follows since

$$
|\Gamma(u+i v)| \sim \sqrt{2 \pi} e^{-\pi|v| / 2}|v|^{u-1 / 2}, \quad|v| \rightarrow \infty, \text { uniformly for bounded real } u,
$$

cf. [1]], p. 141, equation 5.11.9; [8], 8.328(1). 
In the following we will use Lemma B.] with the line of integration $H_{\delta}$, where $\delta=\left(t^{1 /(a c)}-b\right) / a$. Therefore,

$$
e_{c}(a, b)(t)=t^{\left(b-t^{1 /(a c)}\right) / a-1} \frac{1}{2 \pi} \int_{-\infty}^{\infty} t^{i x}\left[\Gamma\left(t^{1 /(a c)}-i a x\right) / \Gamma(b)\right]^{c} d x
$$

and after the change of variable $x=a^{-1} t^{1 /(a c)} u$ and putting $A:=(1 / c+b-a) / a$ we get

$$
e_{c}(a, b)(t)=t^{A-a^{-1} t^{1 /(a c)}} \frac{1}{2 \pi a} \int_{-\infty}^{\infty} t^{i u a^{-1} t^{1 /(a c)}}\left[\Gamma\left(t^{1 /(a c)}(1-i u)\right) / \Gamma(b)\right]^{c} d u .
$$

Binet's formula for $\Gamma$ is $([8], 8.341(1))$

$$
\Gamma(z)=\sqrt{2 \pi} z^{z-1 / 2} e^{-z+\mu(z)}, \quad \operatorname{Re}(z)>0,
$$

where

$$
\mu(z)=\int_{0}^{\infty}\left(\frac{1}{2}-\frac{1}{t}+\frac{1}{e^{t}-1}\right) \frac{e^{-z t}}{t} d t, \quad \operatorname{Re}(z)>0 .
$$

Notice that $\mu(z)$ is the Laplace transform of a positive function, so we have the estimates for $z=r+i s, r>0$, that is,

$$
|\mu(z)| \leqslant \mu(r) \leqslant \frac{1}{12 r},
$$

where the last inequality is a classical version of Stirling's formula, thus showing that the estimate is uniform in $s \in \mathbb{R}$.

Inserting this in (B.4), we get after some simplification

$$
e_{c}(a, b)(t)=\frac{(2 \pi)^{c / 2-1}}{a \Gamma(b)^{c}} t^{A-1 /(2 a)} e^{-c t^{1 /(a c)}} \int_{-\infty}^{\infty} e^{c t^{1 /(a c)} f(u)} g_{c}(u) M(u, t) d u,
$$

where

$$
f(u):=i u+(1-i u) \log (1-i u), \quad g_{c}(u):=(1-i u)^{-c / 2}
$$

and

$$
M(u, t):=\exp \left[c \mu\left(t^{1 /(a c)}(1-i u)\right)\right] .
$$

From (B.7) we get $M(u, t)=1+\mathcal{O}\left(t^{-1 /(a c)}\right)$ for $t \rightarrow \infty$, uniformly in $u$. We shall therefore consider the behavior for large $x$ of the integral

$$
\int_{-\infty}^{\infty} e^{x f(u)} g_{c}(u) d u, \quad x=c t^{1 /(a c)}
$$


This is the same integral as was treated in [7], equation (28), leading to

$$
\int_{-\infty}^{\infty} e^{x f(u)} g_{c}(u) d u=(2 \pi / x)^{1 / 2}\left[1+\mathcal{O}\left(x^{-1}\right)\right]
$$

by methods from [13].

For $x=c t^{1 /(a c)}$ we find

$$
\int_{-\infty}^{\infty} \exp \left(c t^{1 /(a c)} f(u)\right) g_{c}(u) d u=\frac{\sqrt{2 \pi}}{\sqrt{c} t^{1 /(2 a c)}}\left[1+\mathcal{O}\left(t^{-1 /(a c)}\right)\right],
$$

hence

$$
e_{c}(a, b)(t)=\frac{(2 \pi)^{(c-1) / 2}}{a \sqrt{c} \Gamma(b)^{c}} \frac{\exp \left(-c t^{1 /(a c)}\right)}{t^{1-(b-1 / 2+1 /(2 c)) / a}}\left[1+\mathcal{O}\left(t^{-1 /(a c)}\right)\right] .
$$

Pro of of The or e m 2.2. We first prove that $\left(s_{n}(a, b)^{c}\right)$ is S-determinate for $a c \leqslant 2$ by Carleman's criterion, cf. [17], p. 20. In fact, from Stirling's formula we have

$$
s_{n}(a, b)^{c /(2 n)}=(\Gamma(n a+b) / \Gamma(b))^{c /(2 n)} \sim(n a / e)^{a c / 2}, \quad n \rightarrow \infty,
$$

so $\sum s_{n}(a, b)^{-c /(2 n)}=\infty$ if and only if $a c \leqslant 2$.

Since Carleman's criterion is only a sufficient condition for S-determinacy, we need to prove that $e_{c}(a, b)$ is $\mathrm{S}$-indeterminate for $a c>2$. We apply the Krein criterion for S-indeterminacy of probability densities concentrated on the half-line, using a version due to H. L. Pedersen given in [9], Theorem 4. It states that if

$$
\int_{K}^{\infty} \frac{\log e_{c}(a, b)\left(t^{2}\right) d t}{1+t^{2}}>-\infty
$$

for some $K \geqslant 0$, then $e_{c}(a, b)$ is $\mathrm{S}$-indeterminate. This version of the Krein criterion is a simplification of a stronger version given in [15]. We shall see that (3.12) holds for $a c>2$.

From Theorem 2.$]$ we see that (B.12) holds for sufficiently large $K>0$ if and only if

$$
\int_{K}^{\infty} \frac{-c t^{2 /(a c)}}{1+t^{2}} d t>-\infty
$$

and the latter holds precisely for $a c>2$. This shows that $\tau_{c}(a, b)$ is $\mathrm{S}$-indeterminate for $a c>2$.

Proof of Theorem [2.3. The proof uses the same ideas as in [7], but since the proof is quite technical, we give the full proof with the necessary modifications. Since we are studying the behavior for $t \rightarrow 0$, we assume that $0<t<1$ so that $\Lambda:=\log (1 / t)>0$. 
We will need integration along vertical lines

$$
V_{\alpha}:=\{\alpha+i y \mid y=-\infty \ldots \infty\}, \quad \alpha \in \mathbb{R},
$$

and we can therefore express ([L.8) as

$$
e_{c}(a, b)(t)=\frac{t^{b / a-1}}{2 \pi i a \Gamma(b)^{c}} \int_{V_{-b}} t^{z / a} \Gamma(-z)^{c} d z .
$$

By the functional equation for $\Gamma$ we get

$$
e_{c}(a, b)(t)=\frac{t^{b / a-1}}{2 \pi i a \Gamma(b)^{c}} \int_{V_{-b}} g(z) \varphi(z) d z,
$$

where we have defined

$$
\varphi(z):=t^{z / a} \Gamma(1-z)^{c}, \quad g(z):=(-z)^{-c}=\exp (-c \log (-z)) .
$$

Note that $\varphi$ is holomorphic in $\mathbb{C} \backslash[1, \infty)$, while $g$ is holomorphic in $\mathbb{C} \backslash[0, \infty)$.

For $x>0$ we define

$$
g_{ \pm}(x):=\lim _{\varepsilon \rightarrow 0+} g(x \pm i \varepsilon)=x^{-c} e^{ \pm i \pi c} .
$$

C a s e 1 . Assume $0<c<1$.

We fix $0<s<1$, choose $0<\varepsilon<\min (s, b)$ and integrate $g(z) \varphi(z)$ over the contour

$$
\begin{aligned}
\mathcal{C}:= & \{-b+i y \mid y=\infty \ldots 0\} \cup[-b,-\varepsilon] \cup\left\{\varepsilon e^{i \theta} \mid \theta=\pi \ldots 0\right\} \\
& \cup[\varepsilon, s] \cup\{s+i y \mid y=0 \ldots \infty\}
\end{aligned}
$$

and get zero by the integral theorem of Cauchy. On the interval $[\varepsilon, s]$ we will use $g=g_{+}$.

Similarly, we get zero by integrating $g(z) \varphi(z)$ over the complex conjugate contour $\overline{\mathcal{C}}$, and now we use $g=g_{-}$on the interval $[\varepsilon, s]$.

Subtracting the second contour integral from the first leads to

$$
\int_{V_{s}}-\int_{V_{-b}}-\int_{|z|=\varepsilon} g(z) \varphi(z) d z+\int_{\varepsilon}^{s} \varphi(x)\left(g_{+}(x)-g_{-}(x)\right) d x=0,
$$

where the integral over the circle is with positive orientation. Note that the two integrals over $[-b,-\varepsilon]$ cancel. Since $0<c<1$, it is easy to see that the just mentioned integral converges to zero for $\varepsilon \rightarrow 0$, and we finally get for $\varepsilon \rightarrow 0$

$$
\begin{aligned}
e_{c}(a, b)(t) & =\frac{t^{b / a-1}}{2 \pi i a \Gamma(b)^{c}} \int_{V_{s}} g(z) \varphi(z) d z+\frac{t^{b / a-1} \sin (\pi c)}{\pi a \Gamma(b)^{c}} \int_{0}^{s} x^{-c} \varphi(x) d x \\
& :=I_{1}+I_{2} .
\end{aligned}
$$


We claim that $I_{1}$ is $o\left(t^{(s+b) / a-1}\right)$ for $t \rightarrow 0$. To see this, we insert the parametrization of $V_{s}$ and get

$$
\begin{aligned}
I_{1} & =\frac{t^{b / a-1}}{2 \pi a \Gamma(b)^{c}} \int_{-\infty}^{\infty}(-s-i y)^{-c} t^{(s+i y) / a} \Gamma(1-s-i y)^{c} d y \\
& =\frac{t^{(s+b) / a-1}}{2 \pi a \Gamma(b)^{c}} \int_{-\infty}^{\infty} e^{-i y \Lambda / a}(-s-i y)^{-c} \Gamma(1-s-i y)^{c} d y,
\end{aligned}
$$

and the integral is $o(1)$ for $t \rightarrow 0$ by Riemann-Lebesgue's lemma because $\Lambda:=$ $\log (1 / t) \rightarrow \infty$.

The substitution $u=x \Lambda$ in the integral in the term $I_{2}$ leads to

$$
I_{2}=\frac{t^{b / a-1} \sin (\pi c)}{\pi a \Gamma(b)^{c}} \Lambda^{c-1} \int_{0}^{s \Lambda} u^{-c} e^{-u / a} \Gamma(1-u / \Lambda)^{c} d u .
$$

We split the integral in (B.16) as

$$
\int_{0}^{s \Lambda} u^{-c} e^{-u / a}\left[\Gamma(1-u / \Lambda)^{c}-1\right] d u+\int_{0}^{\infty} u^{-c} e^{-u / a} d u-\int_{s \Lambda}^{\infty} u^{-c} e^{-u / a} d u .
$$

Calling the three terms $J_{1}, J_{2}, J_{3}$, we have $J_{2}=a^{1-c} \Gamma(1-c)$ and

$$
J_{3}=-a^{1-c} \Gamma(1-c, s \Lambda / a),
$$

where $\Gamma(\alpha, x)$ is the incomplete gamma function with the asymptotics

$$
\Gamma(\alpha, x)=\int_{x}^{\infty} u^{\alpha-1} e^{-u} d u \sim x^{\alpha-1} e^{-x}, \quad x \rightarrow \infty
$$

(cf. [8], 8.357), hence

$$
J_{3}=\mathcal{O}\left(t^{s / a} \Lambda^{-c}\right), \quad t \rightarrow 0 .
$$

Using the digamma function $\Psi=\Gamma^{\prime} / \Gamma$, we get by the mean-value theorem

$$
\Gamma(1-u / \Lambda)^{c}-1=-\frac{u}{\Lambda} c \Gamma(1-\theta u / \Lambda)^{c} \Psi(1-\theta u / \Lambda)
$$

for some $0<\theta<1$, but this implies that

$$
\left|\Gamma(1-u / \Lambda)^{c}-1\right| \leqslant \frac{c u}{\Lambda} M(s), \quad 0<u<s \Lambda,
$$

where

$$
M(s):=\max \left\{\Gamma(x)^{c}|\Psi(x)| \mid 1-s \leqslant x \leqslant 1\right\},
$$

so $J_{1}=\mathcal{O}\left(\Lambda^{-1}\right)$ for $t \rightarrow 0$. 
This gives

$$
\begin{aligned}
I_{2} & =\frac{t^{b / a-1} \sin (\pi c)}{\pi a \Gamma(b)^{c}} \Lambda^{c-1}\left(\mathcal{O}\left(\Lambda^{-1}\right)+a^{1-c} \Gamma(1-c)+\mathcal{O}\left(t^{s / a} \Lambda^{-c}\right)\right) \\
& =\frac{t^{b / a-1} \Lambda^{c-1}}{(a \Gamma(b))^{c} \Gamma(c)}+\mathcal{O}\left(t^{b / a-1} \Lambda^{c-2}\right),
\end{aligned}
$$

where we have used Euler's reflection formula for $\Gamma$. Since finally

$$
I_{1}=o\left(t^{(s+b) / a-1}\right)=\mathcal{O}\left(t^{b / a-1} \Lambda^{c-2}\right),
$$

we see that (2.2) holds.

Cas e 2. Assume $1<c<2$.

The gamma function decays so rapidly on vertical lines $z=\alpha+i y, y \rightarrow \pm \infty$, that we can integrate by parts in (3.15) to get

$$
e_{c}(a, b)(t)=-\frac{t^{b / a-1}}{2 \pi i a \Gamma(b)^{c}} \int_{V_{-1}} \frac{(-z)^{-(c-1)}}{c-1} \frac{d}{d z}\left(t^{z / a} \Gamma(1-z)^{c}\right) d z
$$

\section{Defining}

$$
\varphi_{1}(z):=\frac{d}{d z}\left(t^{z / a} \Gamma(1-z)^{c}\right)=t^{z / a} \Gamma(1-z)^{c}((1 / a) \log t-c \Psi(1-z))
$$

and using the same contour technique as in Case 1 to the integral in (B.18), where now $0<c-1<1$, we get for $0<s<1$ fixed the equality

$$
e_{c}(a, b)(t)=-\frac{t^{b / a-1}}{a \Gamma(b)^{c}}\left(\tilde{I}_{1}+\tilde{I}_{2}\right)
$$

where

$$
\tilde{I}_{1}=\frac{1}{2 \pi i(c-1)} \int_{V_{s}}(-z)^{-(c-1)} \varphi_{1}(z) d z, \quad \tilde{I}_{2}=\frac{\sin (\pi(c-1))}{\pi(c-1)} \int_{0}^{s} x^{-(c-1)} \varphi_{1}(x) d x .
$$

We have $\tilde{I}_{1}=o\left(t^{s / a} \Lambda\right)$ for $t \rightarrow 0$ by Riemann-Lebesgue's lemma, and the substitution $u=x \Lambda$ in the second integral leads to

$$
\begin{aligned}
& \int_{0}^{s} x^{-(c-1)} \varphi_{1}(x) d x \\
= & \Lambda^{c-2} \int_{0}^{s \Lambda} u^{-(c-1)} \varphi_{1}(u / \Lambda) d u \\
= & -(1 / a) \Lambda^{c-1}\left(\int_{0}^{s \Lambda} u^{-(c-1)} e^{-u / a} d u+\int_{0}^{s \Lambda} u^{-(c-1)} e^{-u / a}\left(\Gamma(1-u / \Lambda)^{c}-1\right) d u\right)
\end{aligned}
$$




$$
\begin{aligned}
& -c \Lambda^{c-2} \int_{0}^{s \Lambda} u^{-(c-1)} e^{-u / a} \Gamma(1-u / \Lambda)^{c} \Psi(1-u / \Lambda) d u \\
= & -a^{1-c} \Lambda^{c-1} \Gamma(2-c)+\mathcal{O}\left(\Lambda^{c-2}\right) .
\end{aligned}
$$

Using the equality

$$
\frac{\sin (\pi(c-1))}{(c-1) \pi}\left(-a^{1-c} \Lambda^{c-1} \Gamma(2-c)\right)=-a^{1-c} \frac{\Lambda^{c-1}}{\Gamma(c)}
$$

obtained by Euler's reflection formula, we see that (2.2) holds.

Ca s e 3. Assume $c>2$.

We perform the change of variable $w=(1 / a) \Lambda z$ in (3.15) and assume that $\Lambda>a$. This gives

$$
e_{c}(a, b)(t)=\frac{t^{b / a-1} \Lambda^{c-1}}{[a \Gamma(b)]^{c}} \frac{1}{2 \pi i} \int_{V_{-(b / a) \Lambda}}(-w)^{-c} e^{-w} \Gamma(1-a w / \Lambda)^{c} d w .
$$

Using Cauchy's integral theorem, we can shift the contour $V_{-(b / a) \Lambda}$ to $V_{-1}$ as the integrand is holomorphic in the vertical strip between both paths and exponentially small at both extremes of that vertical strip. For the holomorphic function $h(z)=$ $\Gamma(1-z)^{c}$ in the domain $G=\mathbb{C} \backslash[1, \infty)$, which is star-shaped with respect to zero, we have

$$
h(z)=h(0)+z \int_{0}^{1} h^{\prime}(u z) d u, \quad z \in G,
$$

hence

$$
\Gamma(1-a w / \Lambda)^{c}=1-\frac{c a w}{\Lambda} \int_{0}^{1} \Gamma(1-u a w / \Lambda)^{c} \Psi(1-u a w / \Lambda) d u
$$

Defining

$$
R(w)=\int_{0}^{1} \Gamma(1-u a w / \Lambda)^{c} \Psi(1-u a w / \Lambda) d u,
$$

we get

$$
\begin{aligned}
& \frac{1}{2 \pi i} \int_{V_{-1}}(-w)^{-c} e^{-w} \Gamma(1-a w / \Lambda)^{c} d w \\
& \quad=\frac{1}{2 \pi i} \int_{V_{-1}}(-w)^{-c} e^{-w} d w+\frac{a c / \Lambda}{2 \pi i} \int_{V_{-1}}(-w)^{1-c} e^{-w} R(w) d w
\end{aligned}
$$

For any $w \in V_{-1}, 0 \leqslant u \leqslant 1$ and for $\Lambda \geqslant a$ it follows that $1-u a w / \Lambda$ belongs to the closed vertical strip located between the vertical lines $V_{1}$ and $V_{2}$. Because 
$\Gamma(z)^{c} \Psi(z)$ is continuous and bounded in this strip, $R(w)$ is bounded for $w \in V_{-1}$ by a constant independent of $\Lambda \geqslant a$. Furthermore, $(-w)^{1-c} e^{-w}$ is integrable over $V_{-1}$ because $c>2$.

On the other hand, in the integral

$$
\frac{1}{2 \pi i} \int_{V_{-1}}(-w)^{-c} e^{-w} d w
$$

the contour $V_{-1}$ may be deformed to a Hankel contour

$$
\begin{aligned}
\mathcal{H}:= & \{x-i \mid x=\infty \ldots 0\} \cup\left\{e^{i \theta} \mid \theta=-\pi / 2 \ldots-3 \pi / 2\right\} \\
& \cup\{x+i \mid x=0 \ldots \infty\}
\end{aligned}
$$

surrounding $[0, \infty)$, and the integral over $\mathcal{H}$ is Hankel's integral representation of the reciprocal gamma function:

$$
\frac{1}{2 \pi i} \int_{\mathcal{H}}(-w)^{-c} e^{-w} d w=\frac{1}{\Gamma(c)} .
$$

Therefore, when we join everything, we obtain for $c>2$ :

$$
e_{c}(a, b)(t)=\frac{t^{b / a-1}}{[a \Gamma(b)]^{c}} \frac{[\log (1 / t)]^{c-1}}{\Gamma(c)}+\mathcal{O}\left(t^{b / a-1}[\log (1 / t)]^{c-2}\right), \quad t \rightarrow 0 .
$$

Cas e 4. $c=1, c=2$.

These cases are easy since $e_{1}(a, b)(t)$ is explicitly given by ([L.5) and $e_{2}(a, b)(t)$ by ([L.15). The asymptotics of $K_{0}$ is known:

$$
K_{0}(t)=\log (2 / t)+\mathcal{O}(1), \quad t \rightarrow 0 .
$$

REMARK 3.1. The behavior of $e_{c}(a, b)(t)$ for $t \rightarrow 0$ can be obtained from (3.14) by using the residue theorem when $c$ is a natural number. In fact, in this case $\Gamma(-z)^{c}$ has a pole of order $c$ at $z=0$, and a shift of the contour $V_{-1}$ to $V_{s}$, where $0<s<1$, has to be compensated by a residue, which will give the behavior for $t \rightarrow 0$.

When $c$ is a natural number, one can actually express $e_{c}(a, b)(t)$ in terms of Meijer's $G$-function:

$$
e_{c}(a, b)(t)=\frac{t^{b / a-1}}{a \Gamma(b)^{c}} G_{0, c}^{c, 0}\left(\begin{array}{llll}
t^{1 / a} \mid & - & \cdots & - \\
& 0 & \cdots & 0
\end{array}\right)
$$

cf. Section 9.3 in [8]. 


\section{ONE-PARAMETER EXTENSION OF THE GUMBEL DISTRIBUTIONS}

The group isomorphism $x=\log (1 / t)$ of the multiplicative group $(0, \infty)$ onto the additive group $\mathbb{R}$ transforms the convolution semigroup $\left(\tau_{c}(a, b)\right)_{c>0}$ into an ordinary convolution semigroup $\left(G_{c}(a, b)\right)_{c>0}$ of probability measures on $\mathbb{R}$ with densities given by

$$
g_{c}(a, b)(x)=e^{-x} e_{c}(a, b)\left(e^{-x}\right), \quad x \in \mathbb{R},
$$

and $a, b, c>0$ are arbitrary. For $c=1$ we have

$$
g_{1}(a, b)(x)=\frac{1}{a \Gamma(b)} \exp \left(-b x / a-e^{-x / a}\right), \quad x \in \mathbb{R} .
$$

This density is infinitely divisible and the uniquely determined convolution roots are given by (4.]).

The special density $g_{1}(a, 1)(x)$ is the Gumbel density with scale parameter $a>0$, and the basic case $a=1$ is discussed in [ $[7]$. From the asymptotic behavior of $e_{c}(a, b)$ in Theorems 2.1 and 2.3 we can obtain the asymptotic behavior of the convolution roots $g_{c}(a, b)$ :

$$
g_{c}(a, b)(x)=\frac{(2 \pi)^{(c-1) / 2}}{a \sqrt{c} \Gamma(b)^{c}} \frac{\exp \left(-c e^{-x /(a c)}\right)}{\exp (x(b-1 / 2+1 /(2 c)) / a)}[1+\mathcal{O}(\exp (x /(a c)))]
$$

for $x \rightarrow-\infty$, and

$$
g_{c}(a, b)(x)=\frac{\exp (-b x / a) x^{c-1}}{[a \Gamma(b)]^{c} \Gamma(c)}+\mathcal{O}\left(\exp (-b x / a) x^{c-2}\right), \quad x \rightarrow \infty .
$$

THEOREM 4.1. All densities $g_{c}(a, b)$ belong to determinate Hamburger moment problems.

Proof. We first prove that $g_{1}(a, b)$ is determinate, and for this it suffices to verify that the moments

$$
s_{n}=\int_{-\infty}^{\infty} x^{n} g_{1}(a, b)(x) d x
$$

satisfy Carleman's condition $\sum_{n=0}^{\infty} s_{2 n}^{-1 /(2 n)}=\infty$ (cf. [17], p. 19). From (4.5)) we get

$$
\begin{aligned}
s_{2 n} & =\frac{1}{a \Gamma(b)} \int_{0}^{\infty}(\log t)^{2 n} t^{b / a-1} \exp \left(-t^{1 / a}\right) d t=\frac{1}{\Gamma(b)} \int_{0}^{\infty}(a \log s)^{2 n} s^{b-1} e^{-s} d s \\
& <\frac{a^{2 n}}{\Gamma(b)}\left(\int_{0}^{1}(\log s)^{2 n} s^{b-1} d s+\int_{1}^{\infty} s^{2 n+b-1} e^{-s} d s\right) .
\end{aligned}
$$


By changing variables we see that

$$
\int_{0}^{1}(\log s)^{2 n} s^{b-1} d s=\frac{(2 n) !}{b^{2 n+1}}
$$

and

$$
\int_{1}^{\infty} s^{2 n+b-1} e^{-s} d s<\Gamma(2 n+b)
$$

hence

$$
s_{2 n}^{1 /(2 n)}<\frac{a}{\Gamma(b)^{1 /(2 n)}}\left[\left(\frac{(2 n) !}{b^{2 n+1}}\right)^{1 /(2 n)}+\Gamma(2 n+b)^{1 /(2 n)}\right],
$$

and the Carleman condition follows from Stirling's formula, which shows that the right-hand side is bounded by $K n$ for sufficiently large $K>0$. We next use Corollary 3.3 in [2] to infer that the Carleman condition also holds for all convolution roots $g_{c}(a, b)$.

Concerning the moments

$$
s_{n}(c)=\int_{-\infty}^{\infty} x^{n} g_{c}(a, b)(x) d x, \quad n \in \mathbb{N}_{0},
$$

of the convolution roots we have the following result:

THEOREM 4.2. The moment $s_{n}(c)$ of (4.6) is a polynomial

$$
s_{n}(c)=\sum_{k=1}^{n} a_{n, k} c^{k}, \quad n \geqslant 1
$$

of degree at most $n$ in the variable $c$. The coefficients $a_{n, k}$ are given below.

Pro of. From (ㅁ.7) we get

$$
\int_{-\infty}^{\infty} e^{-i x y} d G_{c}(a, b)(x)=\int_{0}^{\infty} t^{i y} e_{c}(a, b)(t) d t=[\Gamma(b+i a y) / \Gamma(b)]^{c},
$$

which shows that the negative definite function $\rho$ corresponding to the convolution semigroup $\left(G_{c}(a, b)\right)_{c>0}$ is

$$
\rho(y)=\log \Gamma(b)-\log \Gamma(b+i a y), \quad y \in \mathbb{R} .
$$

The derivatives of $\rho$ can be expressed in terms of the digamma function $\Psi$, namely

$$
\rho^{(n+1)}(y)=-(i a)^{n+1} \Psi^{(n)}(b+i a y), \quad n \in \mathbb{N}_{0},
$$


so if for $n \in \mathbb{N}_{0}$ we define (cf. [3], equation (2.7))

$$
\sigma_{n}:=-i^{n+1} \rho^{(n+1)}(0)=(-a)^{n+1} \Psi^{(n)}(b),
$$

we find

$$
\begin{aligned}
\sigma_{0} & =a \gamma+\frac{a}{b}-a b \sum_{k=1}^{\infty} \frac{1}{k(b+k)}, \\
\sigma_{n} & =a^{n+1} n ! \sum_{k=0}^{\infty} \frac{1}{(b+k)^{n+1}}=a^{n+1} n ! \zeta(n+1, b), \quad n \in \mathbb{N},
\end{aligned}
$$

where $\zeta(z, q)$ is Hurwitz' zeta function (cf. [8], 9.521).

According to [3] we have $s_{1}(c)=\sigma_{0} c, s_{2}(c)=\sigma_{1} c+\sigma_{0}^{2} c^{2}$ and in general $s_{n}(c)$ is given by (4.7), where the coefficients $a_{n, k}$ are determined by the recursion

$$
a_{n+1, k+1}=\sum_{j=k}^{n} a_{j, k}\left(\begin{array}{l}
n \\
j
\end{array}\right) \sigma_{n-j}, \quad n \geqslant k \geqslant 0 .
$$

It is easy to see that

$$
a_{n, 1}=\sigma_{n-1}, \quad a_{n, n-1}=\left(\begin{array}{l}
n \\
2
\end{array}\right) \sigma_{0}^{n-2} \sigma_{1}, \quad a_{n, n}=\sigma_{0}^{n}
$$

\section{REFERENCES}

[1] R. A. Askey and R. Roy, Chapter 5: Gamma Function, in: NIST Handbook of Mathematical Functions, NIST and Cambridge University Press, 2010.

[2] C. Berg, On the preservation of determinacy under convolution, Proc. Amer. Math. Soc. 93 (2) (1985), pp. 351-357.

[3] C. Berg, On infinitely divisible solutions to indeterminate moment problems, in: Proceedings of the International Workshop "Special Functions", Hong Kong, June 21-25, 1999, C. F. Dunkl, M. Ismail, and R. Wong (Eds.), World Scientific, Singapore 2000, pp. 31-41.

[4] C. Berg, On powers of Stieltjes moment sequences. I, J. Theoret. Probab. 18 (4) (2005), pp. 871-889.

[5] C. Berg, On powers of Stieltjes moment sequences. II, J. Comput. Appl. Math. 199 (1) (2007), pp. 23-38.

[6] C. Berg and G. Forst, Potential Theory on Locally Compact Abelian Groups, Ergeb. Math. Grenzgeb., Band 87, Springer, New York-Heidelberg 1975.

[7] C. Berg and J. L. López, Asymptotic behaviour of the Urbanik semigroup, J. Approx. Theory 195 (2015), pp. 109-121.

[8] I. S. Gradshteyn and I. M. Ryzhik, Table of Integrals, Series, and Products, D. Zwillinger (Ed.), eighth edition, Elsevier and Academic Press, Amsterdam 2015.

[9] P. Hörfelt, The moment problem for some Wiener functionals: Corrections to previous proofs, J. Appl. Probab. 42 (3) (2005), pp. 851-860.

[10] Z. J. Jurek, On relations between Urbanik and Mehler semigroups, Probab. Math. Statist. 29 (2) (2009), pp. 297-308. 
[11] G. D. Lin, On powers of the Catalan number sequence, Discrete Math. 342 (7) (2019), pp. 2139-2147.

[12] G. D. Lin and J. Stoyanov, Moment determinacy of powers and products of nonnegative random variables, J. Theoret. Probab. 28 (4) (2015), pp. 1337-1353.

[13] J. L. López, P. Pagola, and E. Pérez Sinusía, A systematization of the saddle point method: Application to the Airy and Hankel functions, J. Math. Anal. Appl. 354 (1) (2009), pp. 347-359.

[14] F. W. J. Olver and L. C. Maximon, Chapter 10: Bessel Functions, in: NIST Handbook of Mathematical Functions, NIST and Cambridge University Press, 2010.

[15] H. L. Pedersen, On Krein's theorem for indeterminacy of the classical moment problem, J. Approx. Theory 95 (1) (1998), pp. 90-100.

[16] R. L. Schilling, R. Song, and Z. Vondraček, Bernstein Functions: Theory and Applications, second edition, Walter de Gruyter \& Co., Berlin 2012.

[17] J. A. Shohat and J. D. Tamarkin, The Problem of Moments, American Mathematical Society, New York 1943.

[18] A. V. Skorokhod, Asymptotic formulas for stable distribution laws, Dokl. Akad. Nauk SSSR (N.S.) 98 (1954), pp. 731-734.

[19] T.-J. Stieltjes, Recherches sur les fractions continues, Ann. Fac. Sci. Toulouse, Math., Sér. 1, 8 (4) (1894), pp. J1-J122.

[20] M. L. Targhetta, On a family of indeterminate distributions, J. Math. Anal. Appl. 147 (2) (1990), pp. 477-479.

[21] Shu-gwei Tyan, The structure of bivariate distribution functions and their relation to Markov processes, Ph.D. Thesis, Princeton University, 1975.

[22] K. Urbanik, Functionals on transient stochastic processes with independent increments, Studia Math. 103 (3) (1992), pp. 299-315.

[23] V. M. Zolotarev, One-dimensional Stable Distributions, American Mathematical Society, Providence, RI, 1986.

\author{
Christian Berg \\ Department of Mathematical Sciences \\ University of Copenhagen \\ Universitetsparken 5 \\ 2100 Copenhagen $\emptyset$, Denmark \\ E-mail: berg@math.ku.dk
}

Received on 10.2.2018,

revised version on 29.6.2018 